\title{
Supraclavicular course of the cephalic vein
}

\author{
Juan David Ramírez, Luis Carlos Sáenz, Diego Rodríguez, \\ Alejandro Jiménez Restrepo, Francisco Villegas
}

\begin{abstract}
Introduction: Vascular access for transvenous pacemaker and cardioverter defibrillator implants is frequently obtained by using the cephalic cutdown technique. Sometimes anatomical variations may limit insertion of one or several leads. We describe a case of a patient with an anomalous supraclavicular course of the left cephalic vein. Case Report: A 61-year-old male with background of ischemic heart disease, hypertension, diabetes mellitus, obstructive sleep apnea and dyslipidemia, was referred to our institution after four months of recurrent syncopal episodes. A bradycardiatachycardia syndrome was diagnosed and decided to proceed with permanent pacemaker implantation. Through a cutaneous incision in the left deltopectoral groove, we dissected the tissue planes until the left cephalic vein became visible. Fluoroscopy in anterior-posterior projection showed clear supraclavicular course of the cephalic vein. This access was abandoned by removing both wires and ligating the proximal
\end{abstract}

Juan David Ramírez ${ }^{1}$, Luis Carlos Sáenz² , Diego Rodríguez ${ }^{3}$, Alejandro Jiménez Restrepo ${ }^{3}$, Francisco Villegas ${ }^{3}$

Affiliations: ${ }^{1} \mathrm{MD}$, Fellow of electrophysiology, Electrophysiology Service, International Arrhythmia Center "Andrea Natale", Fundación Cardioinfantil, Universidad de la Sabana, Bogotá, Colombia; ${ }^{2} \mathrm{MD}$, Chief of Electrophysiology Service, International Arrhythmia Center "Andrea Natale", Fundación Cardioinfantil, Universidad de la Sabana, Bogotá, Colombia; ${ }^{3} \mathrm{MD}$, Electrophysiology Service, International Arrhythmia Center "Andrea Natale", Fundación Cardioinfantil, Universidad de la Sabana, Bogotá, Colombia. Corresponding Author: Dr. Juan David Ramirez, International Arrhythmia Center "Andrea Natale", Street 163 A N ${ }^{\circ} 13 B-$ 60, Bogotá, COLOMBIA - South America. Universidad de la Sabana; Email: juandres2@yahoo.com.ar

Received: 22 October 2013

Accepted: 18 November 2013

Published: 01 April 2014 end of the cephalic vein. Through a fluoroscopy/ venogram guided axillary puncture using the modified Seldinger technique and the retained wire technique, double central vein access was secured, allowing the passage of right atrial and ventricular leads. A dual chamber pacemaker was implanted. Conclusion: Although the supraclavicular course of the cephalic vein is a rare anatomical variant, it is important to recognize its presence as it may lead to potential complications related to lead dysfunction, erosion or collateral vascular damage if used as an access for permanent lead placement. Alternative central vein access is strongly recommended in such cases.

Keywords: Pacemaker, Implantable cardioverter-defibrillator, Cephalic vein, Implanted electrode

\section{How to cite this article}

Ramírez JD, Sáenz LC, Rodríguez D, Restrepo AJ, Villegas F. Supraclavicular course of the cephalic vein. International Journal of Case Reports and Images 2014;5(4):281-284.

doi:10.5348/ijcri-201458-CR-10369

\section{INTRODUCTION}

Vascular access for transvenous pacemaker and cardioverter defibrillator implants is frequently obtained using the cephalic cutdown technique. This method excludes the risk of pneumothorax and subclavian crush syndrome. Despite its benefits, this technique requires surgical dissection skills and there are potential anatomical variations in its course and diameter which may limit or complicate insertion of one or several leads. We describe the case of a patient with an 
anomalous supraclavicular course of the left cephalic vein complicating the implantation of a pacemaker system. We present a review of the available medical literature on this issue.

\section{CASE REPORT}

A 61-year-old male with background of ischemic heart disease with moderate left ventricular impairment, hypertension, diabetes mellitus, obstructive sleep apnea and dyslipidemia, was referred to our institution after four months of recurrent syncopal episodes. A 24-hour Holter monitor documented persistent atrial fibrillation with rapid ventricular response (up to $167 \mathrm{BPM}$ ) alternating with symptomatic bradycardia episodes (29 BPM) and up to six seconds diurnal pauses during atrial fibrillation. As a consequence of the bradycardia-tachycardia syndrome and the requirement for up-titration of beta-blocker therapy permanent pacemaker implantation was needed.

Through a cutaneous incision in the left deltopectoral groove, we dissected the tissue planes until the left cephalic vein became visible. Direct vein punctures allowed for venous access and passage of two hydrophilic wires without difficulty. Fluoroscopy in anteriorposterior projection showed clear supraclavicular course of the cephalic vein (Figure 1). A venogram confirmed anomalous drainage of the left cephalic vein into the ipsilateral external jugular vein. Subsequently, both wires were removed and the terminal portion of the cephalic vein was ligated to achieve hemostasis. Through a fluoroscopy/venogram guided axillary puncture using the modified Seldinger technique and the retained wire technique, double central vein access was secured, allowing the passage of right atrial and ventricular leads, which were subsequently connected to a dual chamber

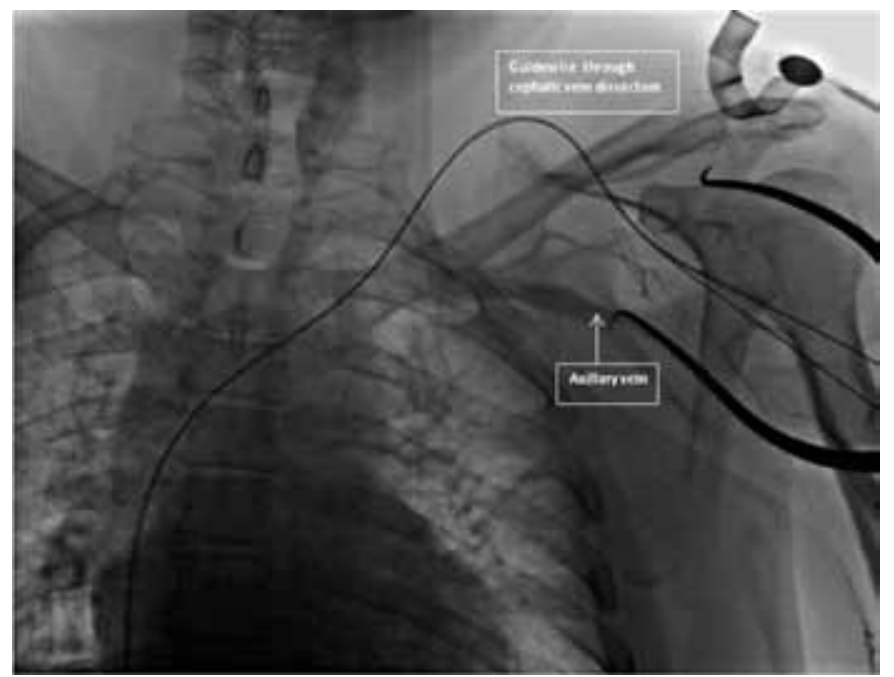

Figure 1: Venography showing the unusual course of the guidewires inserted via the cephalic cutdown technique. Note the supraclavicular trajectory of the guidewires and its spatial relationship with the axillary and subclavian veins, filled with contrast. pacemaker and implanted without complications in a usual pre-pectoral position. The patient was discharged from the hospital the next day after chest X-ray confirmed proper lead placement and device interrogation documented adequate sensing and pacing parameters for both leads (Figure 2). After one week of follow-up, the device was functioning properly and the incision showed normal healing without evidence of infection.

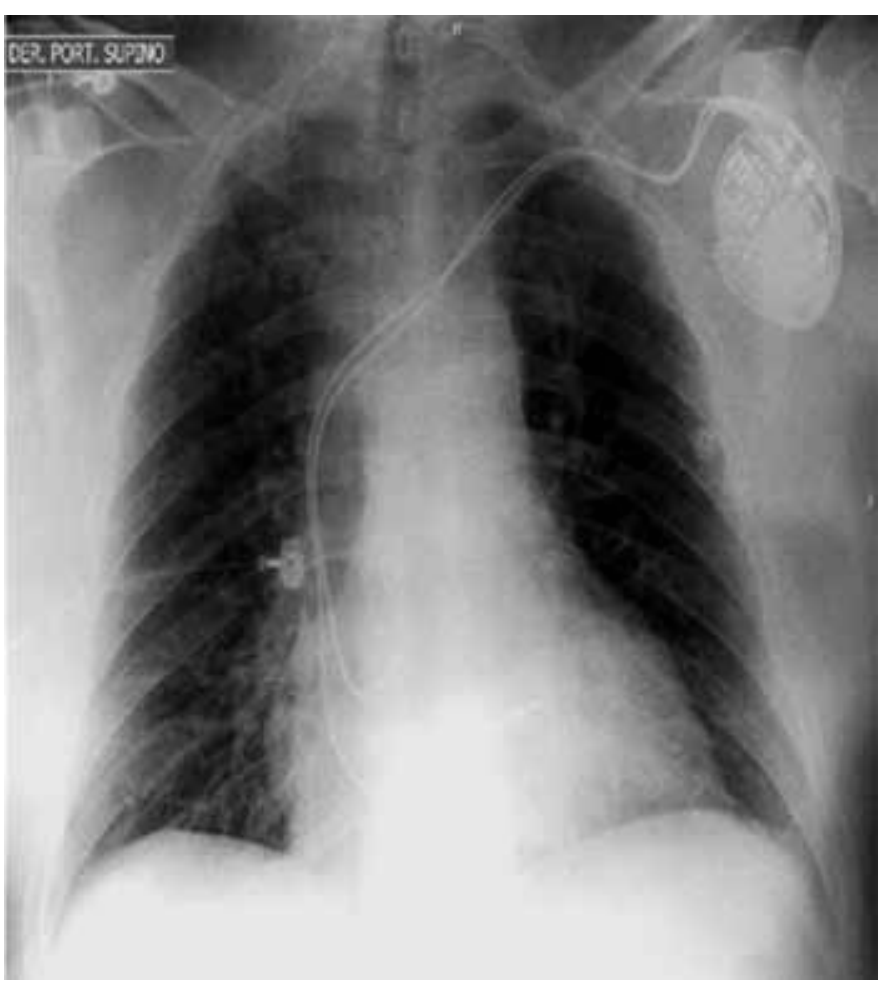

Figure 2: Chest X-ray after pacemaker implantation.

\section{DISCUSSION}

There are two common approaches for cardiac rhythm device leads implantation, epicardial and transvenous. Most leads are placed via the transvenous approach due to the long-term reliability and performance when compared to epicardial leads. The two favored techniques for gaining central venous access are the cutdown technique (commonly employed for the cephalic vein) or the Seldinger venipuncture technique (for axillary or subclavian vein access). Knowledge of the normal venous anatomy of the clavicular and axillary regions, including its potential anatomical variants, is paramount to avoid complications. The preferred access for a cutdown technique approach is the cephalic vein, which is a part of the superficial venous system of the upper extremities [1].

The normal course of the cephalic vein runs along the radial aspect of the forearm, draining blood from the dorsum of the hand. It communicates with the deep venous system of the forearm at the basilic vein. Once 
it crosses the elbow at the antecubital fossa, it moves towards the lateral aspect of the biceps and at the proximal third of the arm dives in between the pectoralis major and deltoid muscles, joining the axillary vein just inferior to the clavicle $[1,2]$. Anatomical studies have documented variations in the course of the cephalic vein, but the clinical cases reported in medical literature are rare, and include cases with absence or small diameter of the cephalic vein, accessory veins running parallel to the cephalic vein or even pre-clavicular or supraclavicular anomalous courses $(<1 \%$ of all dissection series, accounting for less than five reported cases in the medical literature) [3-6]. Only in the case described by Trigano et al. an attempt placing the electrodes was undertaken. However, this case had an anomalous cephalic course draining into the proximal subclavian vein, contrary to our case where a supraclavicular course of the cephalic vein drained into the external jugular vein; the recommendation in such cases is to leave the access and find an alternative approach to avoid lead erosion $[5,6]$. The cephalic vein cutdown is a safe technique to gain central venous access, preventing the well-known complication of subclavian crush syndrome. Its main limitation is the difficulty to separate tissue planes in patients with anatomical deformities or morbid obesity as well as a longer total procedural implant time and the potential need for abandoning access if the vein caliber is small or, less frequently, due to anomalous course (as in this case).

\section{CONCLUSION}

Although the supraclavicular course of the cephalic vein is a rare anatomical variant, it is important to recognize its presence as it may lead to potential complications related to lead dysfunction, erosion or collateral vascular damage if used as an access for permanent lead placement. Alternative central vein access is strongly recommended in such cases.

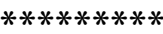

\section{Author Contributions}

Juan David Ramirez - Substantial contributions to conception and design, Acquisition of data, Analysis and interpretation of data, Drafting the article, Revising it critically for important intellectual content, Final approval of the version to be published

Luis Carlos Saenz - Acquisition of data, Revising it critically for important intellectual content, Final approval of the version to be published

Diego Rodríguez - Acquisition of data, Revising it critically for important intellectual content, Final approval of the version to be published

Alejandro Jimenez Restrepo - Acquisition of data, Revising it critically for important intellectual content, Final approval of the version to be published
Francisco Villegas - Acquisition of data, Revising it critically for important intellectual content, Final approval of the version to be published

\section{Guarantor}

The corresponding author is the guarantor of submission.

\section{Conflict of Interest}

Authors declare no conflict of interest.

\section{Copyright}

(C) Juan David Ramirez et al. 2014; This article is distributed under the terms of Creative Commons attribution 3.0 License which permits unrestricted use, distribution and reproduction in any means provided the original authors and original publisher are properly credited. (Please see www.ijcasereportsandimages.com/ copyright-policy.php for more information.)

\section{REFERENCES}

1. Belott PH, Reynolds DW. Permanent pacemaker and implantable cardioverter-defibrillator implantation. In Ellenbogen KA, Kay GN, Lau CP, Wilkoff BL, editors: Clinical Cardiac Pacing, Defibrillation and Resynchronization Therapy, ed 4th, Philadelphia, EEUU, 2011, ElSevier, p. 443-515.

2. Silva MB, Choi L, Chen CC. Peripheral arterial occlusive disease. In Townsend $\mathrm{CM}$, et al, editors: Sabiston Textbook of Surgery: The Biological Basis of Modern Surgical Practice, ed 19th, Philadelphia, EEUU, 2012, ElSevier, p. 1725-84.

3. Yadav S, Ghosh SK, Anand C. Variations of superficial veins of head and neck. J Anat Soc India 2000;49:612.

4. Vollala VR, Bolla SR, Pamidi N. Important vascular anomalies of face and neck - a cadaveric study with clinical implications. Firat Tip Dergisi 2008;13:1236.

5. Trigano A, D’Ivernois C, Levy S, Hartung O, Alimi Y. Preclavicular route following cephalic venous cutdown for pacemaker or defibrillator lead implantation. Pacing Clin Electrophysiol 2007;30(1):147-9.

6. Lau EW, Liew R, Harris S. An unusual case of the cephalic vein with a supraclavicular course. Pacing Clin Electrophysiol 2007;30(5):719-20. 


\section{ABOUT THE AUTHORS}

Article citation: Ramírez JD, Sáenz LC, Rodríguez D, Restrepo AJ, Villegas F. Supraclavicular course of the cephalic vein. International Journal of Case Reports and Images 2014;5(4):281-284.

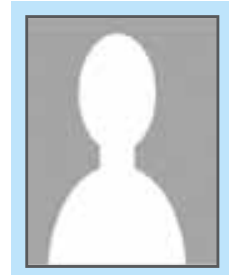

Juan David Ramírez is a fellow of electrophysiology in International Center of Arrhythmias "Andrea Natale" - Universidad de la Sabana, Bogotá- Colombia. His interests are atrial fibrillation and the ventricular arrhythmias. Email juandres2@yahoo.com.ar.

Access full text article on other devices

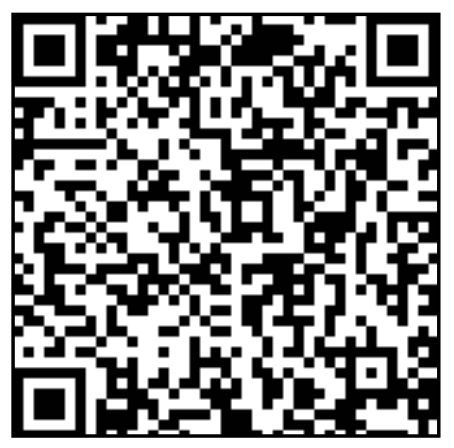

Access PDF of article on other devices

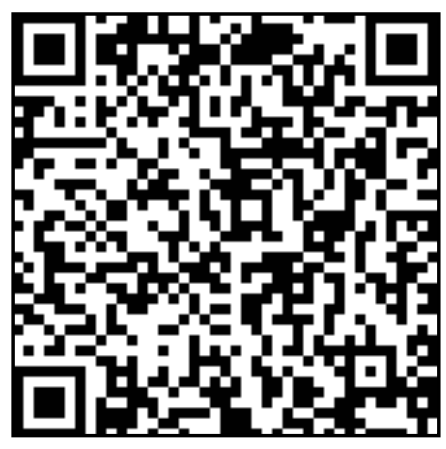

